\title{
L'évaluation des politiques communautaires : légitimation et apprentissages nationaux
}

\author{
Laurence Jourdain
}

\section{(2) OpenEdition \\ 12 Journals}

Édition électronique

URL : http://journals.openedition.org/conflits/387

DOI : $10.4000 /$ conflits.387

ISSN : $1777-5345$

Éditeur :

CCLS - Centre d'études sur les conflits lilberté et sécurité, L'Harmattan

Édition imprimée

Date de publication : 15 janvier 1997

ISSN : 1157-996X

Référence électronique

Laurence Jourdain, «L'évaluation des politiques communautaires : légitimation et apprentissages nationaux », Cultures \& Conflits [En ligne], 28 | hiver 1997, mis en ligne le 16 mars 2006, consulté le 30 mars 2021. URL : http://journals.openedition.org/conflits/387 ; DOI : https://doi.org/10.4000/conflits 387

Ce document a été généré automatiquement le 30 mars 2021.

Creative Commons License 


\title{
L'évaluation des politiques communautaires : légitimation et apprentissages nationaux
}

\author{
Laurence Jourdain
}

Laurence JOURDAIN ${ }^{1}$

2 A l'occasion d'un séminaire organisé par la cellule de prospective de la Commission Européenne, le rapporteur général du $\operatorname{CSE}^{2}$ constatait que " le développement de l'évaluation [répondait] à un ensemble de problèmes étroitement imbriqués : difficultés budgétaires, crise de légitimité de l'action publique, complexité des politiques et interpénétration des niveaux de gouvernement, dysfonctionnements dans les services publics, etc "3. On pourrait néanmoins ajouter que l'évaluation n'est pas seulement une " réponse " apportée à un " problème " objectif; c'est un mode de régulation parmi d'autres, qui n'a rien d'universel, mais qui correspond à un certain type de culture et à un certain modèle institutionnel (on pensera surtout à la gestion des politiques sociales dans les Etats-Unis des années 60). Cela dit, comme beaucoup de pratiques, l'évaluation peut se transmettre, s'exporter ou s'imiter ; son développement actuel dans la plupart des pays d'Europe (et notamment en France) s'explique aussi par des facteurs exogènes. L'influence du cadre communautaire semble en l'occurrence non négligeable. Tout se passe comme si l'émergence à Bruxelles d'une autorité publique vouée à produire des " politiques " dans des secteurs toujours plus diversifiés avait accru la demande d'évaluation à tous les niveaux. Les instances communautaires publient ou suscitent la publication d'un nombre croissant de rapports destinés à mesurer, à capitaliser et à afficher les " impacts " de leurs programmes. Associés d'une façon ou d'une autre à cette pratique, les acteurs nationaux sont amenés à l'intégrer, ou tout du moins à en accepter le principe : la Communauté européenne suscite ou conforte au sein des Etats membres une tendance au " pilotage ex post " (" par les conséquences ") de l'action gouvernementale ${ }^{4}$. Ce phénomène n'est pas anodin ; il importe d'en analyser l'ampleur et la signification. Peut-on parler en matière d'évaluation d'un " modèle communautaire ", susceptible de s'imposer à l'échelon national? Mais pourquoi 
l'évaluation a-t-elle trouvé à Bruxelles un terrain si fertile? Quels sont les critères retenus pour juger du succès ou de l'échec des politiques mises en œuvre, et dans quel système de valeurs s'inscrivent-ils? Qui est compétent pour porter un jugement, qui s'agit-il de convaincre et pour quoi faire ? A partir de là, existe-t-il réellement un " transfert " et une convergence des pratiques au sein des Etats membres? En somme, l'évaluation peut nous servir de " révélateur " ou de " reflet ": classiquement considérée comme une phase, une " séquence " parmi d'autres dans le déroulement des programmes publics ${ }^{5}$, elle offre ici un angle d'approche privilégié pour comprendre la nature du système politique qui est en train de se mettre en place autour de Bruxelles et sa capacité à peser sur les systèmes nationaux... Après avoir mis en évidence les controverses initiales suscitées par l'évaluation à l'échelon national (notamment en France), on s'interrogera donc sur le sens et la fonction des évaluations communautaires, ainsi que sur le degré de diffusion du modèle bruxellois. L'évaluation des politiques publiques : une notion ambiguë et controversée Avant d'essayer d'établir des comparaisons ou des corrélations, il importe de revenir sur la signification du terme " évaluation ": est-ce que tous les acteurs concernés parlent en l'occurrence de la même chose ? De fait, la notion est ambiguë et laisse place à des usages multiples. Le verbe " évaluer " est apparu dans le langage français entre le XIIIe et le XIVe siècle, pour exprimer l'action de " porter un jugement sur la valeur ou sur le prix d'une chose ". Par la suite, il a été alternativement considéré comme synonyme d'expertiser, chiffrer, calculer, ou, au contraire, de fixer approximativement " à vue d'œil ". On ne peut donc pas savoir a priori si l'évaluation suppose ou non une démarche scientifique, quantitative et précise. Quoi qu'il en soit, l'évaluation renvoie forcément au concept de " valeur ", c'est à dire à une idée partagée de ce qui est vrai, beau, bon ou désirable à l'intérieur d'une collectivité sociale déterminée ${ }^{6}$. Ceci confère à l'action d'évaluer un caractère contingent et relatif. Mais il semblerait que notre vocabulaire soit plus restrictif que celui des pays anglo-saxons : comme le soulignait un rapport de l'OCDE, ce que nous appelons évaluation peut alternativement se traduire par " review ", " examination ", " assessment ", " survey ", " accounting "7... L'évaluation pourrait donc s'affranchir de son rapport étymologique aux valeurs pour se dissoudre dans une vaste gamme d'activités allant de l'inspection et de l'examen critique à la mise en jeu d'une responsabilité. A partir de là, il était logique que l'évaluation des " politiques publiques ", des actions des autorités publiques au sein de la société, ne fasse pas l'objet d'un consensus. Dans la plupart des démocraties libérales, on voit effectivement s'affirmer des conceptions divergentes: l'approche " rationaliste " s'oppose à l'approche " pluraliste ", le " positivisme " au " constructivisme ", la méthode quantitative à la méthode qualitative, l'évaluation " managériale " à l'évaluation " démocratique ". Ces différentes définitions se retrouvent en France, où elles se sont successivement imposées. Pour Michel Deleau ${ }^{8}$ ou pour Jean-Pierre Nioche ${ }^{9}$, l'évaluation a pour objectif d'" apprécier et mesurer les effets propres d'une action gouvernementale " à l'aide d'indicateurs quantitatifs; sa qualité est alors liée à sa reproductibilité (voire à sa falsifiabilité) et à son objectivité (assurée par l'indépendance et la compétence scientifique des évaluateurs). Pour Patrick Viveret et pour les héritiers de la gauche " moderniste " ou de certains courants auto-gestionnaires ${ }^{10}$, l'évaluation doit plutôt être un " jugement sur la valeur de l'action politique ", c'est à dire un gage de transparence au service des citoyens, en même temps qu'un instrument de " management " destiné à améliorer l'efficacité du fonctionnement administratif. Pour Eric Monnier ${ }^{11}$, l'action d'évaluer consiste plus précisément à " juger de la valeur d'une action, au regard de 
critères explicites et sur la base d'informations ad hoc ", mais en prenant en compte la pluralité des acteurs, des valeurs et des enjeux qui sous-tendent les politiques publiques, ainsi que les " dérives " subies par les objectifs initiaux des programmes au gré de leur mise en œuvre ; l'évaluation est par nature " contingente "; elle ne peut être crédible et utile que si elle se conçoit comme un processus d'apprentissage collectif et d'analyse négociée des données. La plupart des auteurs considèrent néanmoins que les définitions pluralistes et démocratiques de l'évaluation n'ont vraiment trouvé leur application que dans les pays nordiques ou anglo-saxons: la France resterait prisonnière d'une approche rationaliste, à cause de la survivance d'une idéologie de l'intérêt général et d'une tradition jacobine, du monopole de l'expertise légitime exercé par les grands corps de l'Etat, de la confusion persistante entre " évaluation " et " contrôle administratif ", ou encore de la domination exercée par les sciences expérimentales dans le champ scientifique ${ }^{12}$. En réalité, la conception française est désormais plus nuancée ; le rationalisme absolu qui s'incarnait à la fin des années 1960 dans des méthodes liées à la planification, comme la " rationalisation des choix budgétaires " (RCB), semble avoir été définitivement abandonné. L'évaluation des politiques publiques a commencé à se développer, dans le cadre notamment des programmes de modernisation du service public lancés par le Gouvernement Rocard ; plusieurs dispositifs sectoriels ont peu à peu été mis en place (du Comité National d'Evaluation de la Politique de la Ville à la Commission Nationale d'Evaluation du Revenu Minimum d'Insertion); un Comité Interministériel, un Fonds National et un Conseil Scientifique de l'Evaluation ont été institués par le décret du 22 janvier $1990^{13}$; les lois du 14 juin 1996 ont enfin prévu la création d'un Office Parlementaire d'Evaluation des Politiques Publiques et d'un Office Parlementaire d'Evaluation de la Législation. Il faut toutefois reconnaître que cette évolution est très récente et qu'elle reste précaire: le Président du CSE constatait dernièrement que " le nombre de rapports officiels produits en France sur l'évaluation ou mentionnant cette vache sacrée (...) [était] en proportion inverse de l'intérêt pratique que le Gouvernement et le Parlement lui accordent concrètement "; il déplorait surtout le " maigre bilan " de la procédure interministérielle mise en œuvre ${ }^{14}$. Quoi qu'il en soit, on a plusieurs façons de définir ce qu'est l'évaluation des politiques publiques et chaque définition entraîne une interprétation spécifique des fonctions, voire des finalités de l'évaluation. Il reste en effet à savoir si le but est de contrôler les dépenses publiques, de répartir plus efficacement les ressources financières et humaines, de moderniser la gestion des programmes ou de l'appareil administratif, de préparer et d'éclairer les choix politiques, de " rendre compte aux citoyens ", ou encore de mobiliser les partenaires concernés autour de la compréhension du processus auquel ils participent. A priori, ces ambiguïtés ne facilitent pas l'analyse... Cela dit, on ne se trouve pas pour autant face à un ensemble de pratiques indigènes, hermétiquement isolées. On constate au contraire que l' " évaluation des politiques publiques " s'est peu à peu consolidée dans l'ensemble des pays industrialisés occidentaux, comme un domaine d'action et comme un " objet " spécifiques. La multiplication des rapports, des colloques et des séminaires internationaux, la mise en place de structures et la constitution de réseaux transfrontaliers de " spécialistes " ont contribué à donner à l'évaluation un contenu, des frontières et une unité relative. Or, une part de ce travail de construction s'est faite à l'échelon européen... Le développement des évaluations communautaires Le développement et l'institutionnalisation de l'évaluation ont été plus précoces à Bruxelles qu'au sein de la plupart des Etats membres : dès le début des années 1970, des 
structures ad hoc ont été mises en place et une réflexion méthodologique s'est esquissée. A l'heure actuelle, l'évaluation est devenue une exigence incontournable, encadrée par des normes juridiques, dans plusieurs secteurs. On peut toutefois s'interroger sur les causes d'un tel essor et sur la nature du " modèle " proposé : quelles sont les conceptions et les pratiques dominantes? Quelles sont leur portée et leurs limites? Si l'évaluation devient un critère pour juger a posteriori ou à mi-parcours de la pertinence et de l'efficacité des actions mises en œuvre, sera-t-elle pour autant utilisée pour améliorer la gestion quotidienne? Servira-t-elle d'argument pour remettre en cause certains choix, ou pour arbitrer entre des intérêts concurrents? Ou bien est-elle avant tout conçue pour conférer aux mécanismes communautaires une meilleure transparence et pour responsabiliser les autorités compétentes? En somme, quelles sont les fonctions réelles de l'évaluation à Bruxelles? Des secteurs privilégiés La pratique de l'évaluation s'est tout d'abord développée dans le cadre la politique de recherche scientifique et technologique communautaire. Progressivement définie à partir des années 1960, cette politique a désormais pour objectif officiel de " renforcer les bases scientifiques et technologiques de l'industrie européenne et de favoriser le développement de sa compétitivité internationale, ainsi que de promouvoir les actions de recherche jugées nécessaires au titre d'autres chapitres du traité "15 ; elle repose sur l'adoption de programmes pluriannuels, qui donnent lieu à la sélection sur appels d'offres, puis au cofinancement de projets exécutés " en partenariat " par les laboratoires et les entreprises des Etats membres. L'évaluation des programmes s'est imposée très tôt, mais elle a toujours été associée à d'autres pratiques, comme la réflexion prospective. Le 14 janvier 1974, le Conseil a par exemple lancé un " premier programme d'action en matière de prévision, d'évaluation et de méthodologie "16, qui devait être régulièrement renouvelé et élargi par la suite ${ }^{17}$. Les années suivantes, la Commission organisait toute une série de séminaires et de colloques sur l'évaluation, associant des experts européens, américains ou japonais. A partir de 1983, des règles précises allaient être fixées ${ }^{18}$ et une unité indépendante allait être chargée de piloter toutes les évaluations menées par la DGXII (" Science, Recherche et Développement "), ainsi que d'animer la réflexion méthodologique en concertation avec les autres services de la Commission. Aujourd'hui, l'évaluation de chaque programme est obligatoire et en conditionne le renouvellement; une enveloppe budgétaire (de l'ordre de 0,5 à $1 \% \mathrm{du}$ budget global) est prévue à cet effet. Si la politique de recherche a en quelque sorte servi de " terrain d'expérimentation "19 et reste toujours " une des plus évaluées "20, le modèle communautaire semble avoir pris une nouvelle dimension en s'étendant à d'autres domaines, comme celui de la cohésion économique et sociale. Destinée à " promouvoir un développement harmonieux de l'ensemble de la Communauté ", et en particulier à " réduire l'écart entre les niveaux de développement des régions ou le retard des régions les moins favorisées, y compris les zones rurales "21, la politique de cohésion permet de cofinancer la réalisation de programmes conçus conjointement par les autorités nationales et régionales, par l'intermédiaire de trois " fonds structurels ": le Fonds Européen de Développement Régional (FEDER), le Fonds Social Européen (FSE) et le Fonds Européen d'Orientation et de Garantie Agricole (FEOGA-Orientation). Depuis la réforme et le regroupement de ces fonds, décidés en 1988 par le Conseil ${ }^{22}$, de nouveaux principes directeurs ont été fixés : concentration des crédits autour de cinq objectifs prioritaires, décentralisation de la mise en œuvre, " additionnalité " des financements communautaires et nationaux, création et consolidation d'un partenariat entre les différents échelons impliqués... et évaluation systématique des actions mises 
en œuvre. Ces impératifs ont été confirmés et précisés pour la période 1994-1999 par les règlements du 20 juillet $1993^{23}$. Désormais, l'évaluation de chaque programme est donc obligatoire (ici encore, un pourcentage budgétaire est prévu à cet effet). Mais elle est menée de façon décentralisée, sous la responsabilité d'un " comité de suivi " composé de représentants de la Commission et de la Banque Européenne d'Investissement, de l'Etat et des collectivités territoriales concernées, voire des acteurs économiques et sociaux. Dans ces deux secteurs, même si elle n'est pas toujours vraiment appliquée dans les faits, l'approche dite " pluraliste " s'est imposée comme un modèle de référence. En matière de recherche, les évaluations sont en principe confiées à des " panels " de 6 à 8 experts extérieurs recrutés au cas par cas par la Commission ${ }^{24}$. Si la tradition de " l'évaluation par les pairs ", axée sur les résultats scientifiques des programmes, était de règle au départ, les responsables bruxellois ont peu à peu admis la nécessité de diversifier les critères d'appréciation et d'élargir les " panels " aux utilisateurs de la recherche (notamment aux représentants industriels). Cette conception, qui apparaissait déjà dans les conclusions des colloques de Milan et de Copenhague (organisés en 1976 et 1979 respectivement), semble aujourd'hui dominante: l'évaluation est de plus en plus souvent présentée comme un processus d'apprentissage et de médiation entre les différents acteurs intéressés, c'est à dire comme une façon de confronter les intérêts et les systèmes de valeurs, de " poursuivre la négociation par d'autres moyens ". Un rapport publié par la Commission en 1990 observait par exemple: " l'évaluation est un processus social qui implique la multiplicité des acteurs dans un dialogue qui est par nature source de clarification et de compréhension mutuelle "25. Dans le cas de la cohésion économique et sociale, les évaluations sont en règle générale réalisées par des organismes extérieurs sélectionnés sur appels d'offres, mais le partenariat et l'analyse collective des données sont nécessaires. La Commission diffuse d'ailleurs des notes d'orientation, avec des recommandations méthodologiques détaillées, qui présentent l'évaluation comme " une analyse permettant de mettre à disposition des autorités et des citoyens des informations sur les résultats de l'action publique "26. Ces recommandations sont souvent répercutées par des organismes nationaux: en France, le Délégué à l'Aménagement du Territoire et à l'Action Régionale précisait par exemple, dans une note aux Préfets de Région de mai 1995 que l'évaluation était " un outil d'aide à la prise de décision, que l'ensemble des partenaires impliqués [devait] s'approprier "; en juin 1996, il leur rappelait que " le partenariat avec les Conseils régionaux, les Conseils généraux et l'ensemble des acteurs économiques et sociaux " restait un principe essentiel et il leur recommandait de " veiller tout particulièrement à la qualité de cette concertation ". L'évaluation des politiques communautaires s'est donc développée, sous une forme " pluraliste " revendiquée, dans les domaines de la recherche et de la cohésion. Toutefois, force est de constater que ce phénomène ne s'est pas manifesté avec la même acuité dans tous les secteurs. A l'heure actuelle, seules quelques Directions Générales de la Commission se sont d'ailleurs dotées d'une unité " évaluation " : en dehors de la DGXII (précédemment évoquée), on peut citer les DGV (" Emploi, relations industrielles et affaires sociales "), VI (" Agriculture ") et XVI (" Politiques régionales "), qui sont respectivement compétentes pour la gestion du FEOGA, du FSE et du FEDER ${ }^{27}$. On notera que la DGVIII, chargée de la coopération avec les pays en voie de développement, s'est également orientée vers une évaluation systématique des actions financées ; il semblerait toutefois que la mesure des impacts soit pour l'instant réalisée " projet par projet " et confiée aux organisations non gouvernementales ou aux experts 
chargés de la mise en œuvre sur le terrain, sans qu'une perspective " horizontale " ne $s^{\prime}$ impose vraiment ${ }^{28}$. Quoi qu'il en soit, l'évaluation ne s'est institutionnalisée que dans quelques types très particuliers de politiques communautaires: on peut dès lors se demander ce que ces politiques ont en commun et pourquoi elles se sont révélées plus propices que d'autres à l'enracinement d'une telle pratique... Un contexte d'incertitude Les actions menées dans les domaines de la recherche et de la cohésion économique et sociale (ainsi d'ailleurs que la coopération avec les pays en voie de développement) supposent l'attribution de crédits, de prestations ou d'avantages à certaines catégories de bénéficiaires. Elles pèsent sur le budget communautaire dans des proportions variables, mais au fond, si on laisse de côté la politique agricole commune, il s'agit des rares secteurs dans lesquels l'intervention bruxelloise ne se borne pas à produire du droit. L'Europe sort ici de son rôle " régulateur " et ces modestes concessions à l'idée d' " Etat Providence " ou d'" Etat keynésien " pourraient bien être le " prix à payer " pour que les contraintes de l'intégration soient acceptées ${ }^{29}$. La Communauté n'apporte toutefois qu'un " cofinancement " sur la base de programmes à durée limitée, dont la reconduction n'est en principe pas automatique. Elle s'efforce par ailleurs d'agir "à la marge " et de favoriser l'émergence de projets structurants : les politiques considérées se veulent incitatives et contractuelles. On se trouve donc face à un très fort degré d'incertitude. La pertinence d'une évaluation systématique semble dès lors doublement justifiée. Pourtant, il n'est pas certain que les évaluations réalisées jusqu'à présent aient réellement contribué à réduire l'incertitude attachée aux impacts des actions mises en œuvre, ni qu'elles aient permis de réorienter les décisions politiques ou les pratiques administratives. Il pourrait être intéressant à cet égard de disséquer les différents rapports publiés depuis une dizaine d'années... Pour l'instant, à titre d'illustration, on se contentera d'évoquer le cas de la recherche scientifique et technologique, où le décalage entre les objectifs officiels et les effets obtenus, entre les arguments utilisés au moment des négociations intergouvernementales et les critères retenus dans les évaluations, est particulièrement flagrant. L'affichage d'un " bilan globalement positif " va en effet de pair avec l'aveu d'une incapacité à " évaluer (...) la transformation des progrès scientifiques et technologiques en avantages économiques "130. Les rapports se focalisent donc essentiellement sur des résultats " académiques " (nombre de thèses soutenues, d'articles publiés, etc). Par ailleurs, le pouvoir incitatif des actions est controversé et " l'effet d'aubaine " semble important : une étude réalisée par le Centre de Sociologie de l'Innovation de l'Ecole des Mines constatait par exemple que " l'action de la Commission [infléchissait] très peu les thématiques " et que pour 70 $\%$ des équipes financées, les contrats communautaires auraient surtout permis d'accélérer des projets en cours ${ }^{31}$. Selon les évaluateurs du programme de recherche ESPRIT $^{32}, 20 \%$ des entreprises participantes auraient " de toute manière réalisé ces travaux " et 50 \% l'auraient fait, " mais avec une ampleur inférieure " ${ }^{33}$. Les intéressés eux-mêmes reconnaissent ce fait : " le CNRS ne va pas changer sa politique pour passer à Bruxelles, c'est clair "; " quand les laboratoires ont un projet, on voit si on peut le faire financer par la Communauté, ou ailleurs " ${ }^{34}$. Mais, le discours dominant justifie ces incertitudes et se contente de " supposer " l'existence d'une évolution: " ce n'est évidemment qu'à moyen et à long terme que pourront être vérifiés les résultats de cet ambitieux système d'équations " 35 ; " il est trop tôt pour évaluer l'impact économique de ces initiatives; on peut tout au plus estimer qu'elles sont positives en ce qu'elles permettent de multiplier les chances de réussite et de réduire les risques " ${ }^{36}$. On constate également l'existence d'un décalage entre la diffusion très large des rapports 
et leur faible impact sur la gestion quotidienne ou sur la programmation : une étude publiée par la Commission admettait ainsi qu'en matière de recherche, les recommandations finales étaient peu appliquées, tout en imputant ces limites à des problèmes de calendrier ${ }^{37}$. En tout état de cause, les évaluations bruxelloises se focalisent apparemment sur le succès des programmes plus que sur leurs échecs et les seules vraies critiques portent en règle générale sur la lourdeur des procédures: le bien-fondé de l'intervention elle-même ne risque donc pas d'être remis en question. Certains des " bénéficiaires " considèrent d'ailleurs ces évaluations comme des alibis justifiant la reconduction systématique des programmes existants: " les gens de la DGXII et de la DGXIII soutiennent des programmes qui se perpétuent pour eux-mêmes et ne se posent jamais vraiment la question de leur utilité réelle "; " depuis dix ans, aucun programme n'a jamais disparu, la plupart sont toujours gérés, exécutés et évalués par les mêmes personnes "; " à partir du moment où il existe des services créés exprès, des gens qui en vivent, un programme peut continuer éternellement, même s'il ne sert à rien, tant qu'il ne dérange personne "; " de toute façon la Communauté n'a pas les moyens nécessaires pour être sélective "... ${ }^{38}$ Les agents de la Commission restent quant à eux très prudents : " l'évaluation n'a pas à décider de la continuation ou de l'arrêt d'un programme ", mais elle " apporte un ensemble d'informations qui, combinées avec d'autres, permettent aux responsables politiques de prendre (en toute liberté) des décisions qui relèvent de la nature de leurs fonctions "39. Si les politiques menées par la Communauté dans les secteurs de la recherche et de la cohésion économique et sociale (ou de la coopération au développement) se caractérisent par leur coût relatif et par l'incertitude qui entourent leurs effets, elles ont toutefois un autre point commun : ces trois politiques n'étaient en effet pas explicitement prévues par les traités fondateurs et elles se sont imposées progressivement, en dépit de la résistance manifestée par les Etats membres (qui entendaient en l'occurrence conserver leurs compétences et leur autonomie) ${ }^{40}$. On peut dès lors se demander si les évaluations menées à Bruxelles n'ont pas pour fonction première de légitimer l'autorité et l'intervention communautaires... Un nouveau mode de légitimation? La légitimité des autorités communautaires ne fait pas l'objet d'un consensus et se heurte depuis l'adoption du traité sur l'Union européenne à une contestation renouvelée. La question des pouvoirs dévolus au Parlement de Strasbourg est en l'occurrence contingente, dans la mesure où la plupart des Assemblées nationales ne remplissent plus qu'en apparence leurs fonctions traditionnelles (et notamment celle qui consiste à " faire les lois "); le vrai problème réside dans le fait que le système communautaire ne fonctionne pas selon les règles, actuellement présentées comme " universelles ", de la démocratie représentative et pluraliste. Au sein des quinze Etats membres de l'Union, la légitimité du pouvoir politique se fonde avant tout sur les modes de désignation et de révocation de ceux qui l'exercent : les gouvernants ne peuvent accéder et se maintenir au pouvoir, qu'avec la confiance du peuple, et/ou de l'Assemblée élue par le peuple. A Bruxelles, les critères sont brouillés, dans la mesure où rien ne permet véritablement aux autorités qui produisent des règles de droit et des politiques publiques d'en assumer la responsabilité directe ; l'élection au suffrage universel des députés européens, le droit de censure qui pèse en théorie sur la Commission et la faculté qu'ont les quinze Parlements nationaux de révoquer leurs Gouvernements (donc leurs représentants au Conseil) ne font guère illusion. L'opacité du processus décisionnel et l'absence de réelle séparation des pouvoirs interdisent de facto aux gouvernés de participer à la définition des politiques communautaires ou d'en contrôler les auteurs. Or, aucun pouvoir ne 
peut exister sans un minimum de consentement et d'obéissance de la part des agents sociaux sur lesquels il s'exerce : faute d'incarner " l'expression de la volonté générale " d'un peuple ou d'une nation, les interventions communautaires seront justifiées par leurs résultats et par leur efficacité ; la question de savoir " qui gouverne " et " au nom de qui " sera éclipsée par la mise en valeur d'un pouvoir " fonctionnel ". En s'engageant à démontrer régulièrement l'efficacité de son action, en reconnaissant un pouvoir de jugement à des personnalités choisies pour leur compétence et/ou pour leur représentativité, la Commission semble avoir trouvé un moyen de mettre en jeu sa responsabilité ou, tout du moins, de proposer un substitut, une alternative aux mécanismes traditionnels de la démocratie parlementaire. Le développement sélectif de l'évaluation à Bruxelles ne correspond donc pas seulement à des impératifs gestionnaires : ce qui est en jeu, c'est une certaine idée de l'autorité communautaire et de sa vocation à agir au sein de la société. Mais on ne peut pas non plus se contenter d'évoquer une " crise de légitimité de l'action publique " au sens large : si le bien-fondé des politiques menées à l'échelon européen est régulièrement remis en cause, c'est aussi au nom de la souveraineté. Comme dans toutes les grandes organisations internationales, l'évaluation remplit alors son rôle de " production d'information structurée pour répondre à une attente ou induire un jugement de valeur " ; " elle n'est en rien orientée vers l'amélioration des projets, mais vers le renforcement des codes et des normes de l'organisation, vers l'adoption et la diffusion d'une culture technique " 41 . L'évaluation systématique des politiques communautaires les plus coûteuses et les plus incertaines ne constitue bien évidemment qu'une solution partielle et ne peut tenir lieu de contrôle démocratique. Tout d'abord, comme on l'a vu précédemment, les conclusions et les recommandations publiées n'influent que très partiellement sur la prise de décision; elles ne conditionnent en aucun cas le maintien en fonction des autorités compétentes. D'autre part, les évaluations communautaires ne s'adressent jamais vraiment aux citoyens eux-mêmes : si elles sont très largement diffusées, elles prennent surtout pour " cibles " les décideurs et les clients des actions mises en oeuvre, c'est à dire les acteurs susceptibles de peser sur leur reconduction, en tant que " financeurs " ou en tant que " bénéficiaires ". Dans les secteurs de la recherche ou de la cohésion économique et sociale, les rapports d'évaluation sont effectivement transmis au Conseil et au Parlement Européen, aux administrations nationales, aux centres de recherche, aux entreprises industrielles ou aux instances régionales, aux organisations syndicales et patronales, voire à la presse spécialisée. Des séminaires sont éventuellement organisés pour présenter les résultats aux acteurs les plus impliqués ; les conférences de presse et les articles destinés au " grand public " sont beaucoup plus rares... Cela dit, pour renforcer leur légitimité, les services bruxellois concernés ont aussi intérêt à ce que leur conception de l'évaluation et de son utilité pour l'action publique soit elle-même acceptée et partagée. Or cette conception semble effectivement se diffuser au sein des systèmes nationaux : dans le cas de la France tout du moins, l'émergence récente d'une " culture de l'évaluation " ne s'explique pas entièrement si l'on fait abstraction de l'influence communautaire. Mais jusqu'à quel point cette influence peut-elle être prise en compte? Un phénomène de transfert et d'apprentissage? Appliquées à l'étude des pratiques politiques, les notions de " transfert " ou d' " apprentissage " ne sont pas neutres. Elles trouvent leurs source dans un désir d'expliquer scientifiquement le comportement humain et notamment dans l'héritage des behavioristes américains, qui ont eux mêmes repris les apports de plusieurs disciplines, de la psychologie à la cybernétique. L'" apprentissage " peut donc 
se définir alternativement comme une modification du comportement suscitée par des expériences répétées, ou comme l'aptitude d'un système à améliorer son fonctionnement en s'adaptant à des stimuli extérieurs; le " transfert " serait alors le phénomène par lequel les progrès obtenus au cours de l'apprentissage se répercutent dans un autre domaine d'activité ou dans un autre système. Même si leur usage nécessite un minimum de vigilance, ces notions semblent particulièrement appropriées à l'analyse des relations entre les pratiques communautaires et nationales en matière d'évaluation. Ces relations ne peuvent en effet se décliner qu'en termes d'adaptation progressive : il serait vain d'envisager ici un lien de causalité direct, une contrainte objective ou une métamorphose soudaine; le cas échéant, les normes bruxelloises seront intériorisées, appropriées et reproduites de façon diffuse, par l'intermédiaire d'une multiplicité de canaux. Néanmoins, l'existence d'un phénomène d'apprentissage ou de transfert reste difficile à démontrer. A ce stade, on devra se contenter de rappeler l'antériorité des pratiques communautaires et de mettre en évidence le travail de diffusion entrepris par les services de la Commission, pour analyser à travers quelques exemples la façon dont les agents nationaux s'adaptent, refusent ou anticipent cette mutation. Le prosélytisme de la Commission Quels que soient les résultats escomptés et effectivement atteints, on peut déjà considérer comme acquise l'existence à Bruxelles d'une volonté de diffusion, d'inculcation et de persuasion. Les services concernés de la Commission déploient un zèle manifeste pour amener les agents nationaux à intégrer une " culture de l'évaluation " et, partant, pour se doter de partenaires partageant les mêmes croyances (ou, tout du moins, le même langage) en matière d'action publique. Dans le domaine de la recherche, l'effort porte avant tout sur la mobilisation et la constitution d'un forum d'experts à l'échelle européenne. Dès le milieu des années 1970, la DGXII a sollicité la collaboration et encouragé la mise en réseau d'un certain nombre de structures nationales ${ }^{42}$, autour d'une vaste réflexion sur les méthodes et les indicateurs de l'évaluation scientifique et technologique. A l'origine, les colloques et journées d'études organisés par la Commission constituaient la principale source d'interaction. Avec le lancement des programmes FAST et MONITOR, les partenaires extérieurs ont également pu être associés dans des contrats de recherche et réaliser un certain nombre d'études " transversales " sur l'évaluation. En 1994, enfin, la Commission a suscité la création du réseau ETAN (European Technology Assessment Network), qui est destiné à réunir des gestionnaires de la recherche et des chercheurs nationaux spécialisés dans l'évaluation des politiques scientifiques, la veille technologique ou la prospective. Dans le secteur de la cohésion économique et sociale, l'effort de diffusion semble avoir été encore plus direct. Comme on l'a vu précédemment, la Commission multiplie en effet les incitations, les recommandations et les conseils méthodologiques pour aider les acteurs régionaux et locaux à mener à bien leur propres évaluations, tout en exploitant des relais nationaux (comme le Commissariat au Plan ou la DATAR en France) ${ }^{43}$. En 1990, la Communauté a d'autre part lancé le programme MEANS (Méthodes d'Evaluation des Actions de Nature Structurelle) afin de financer des recherches sur les méthodes d'évaluation, des évaluations-pilotes ou des séminaires de formation; reconduit en 1994, ce programme est actuellement coordonné par la DGXVI et mis en œuvre par un cabinet de conseil rassemblant des " spécialistes " issus de plusieurs Etats membres, le Centre Européen d'Expertise en Evaluation $^{44}$. Des comportements nationaux différenciés Face à la sollicitation multiforme dont ils font l'objet, les agents nationaux réagissent en fonction de leurs intérêts, de leurs valeurs et de leurs ressources respectives. De façon générale, les 
administrations françaises semblent s'être peu à peu adaptées aux exigences bruxelloises; la pratique de l'évaluation est acceptée, parfois réappropriée et étendue au pilotage des politiques nationales. Mais, ce phénomène de transfert reste inégal, et l'apprentissage trouve d'un secteur à l'autre une signification très différente. A l'échelon régional et local, le recours systématique à l'évaluation imposé dans la mise en œuvre des programmes financés par les fonds structurels tend à se généraliser : depuis 1993, il est notamment prévu pour les contrats de plan Etat-région et pour les contrats de ville. Les évaluations impulsées par les collectivités territoriales se multiplient et cette pratique semble éveiller un intérêt croissant de la part des responsables politiques et administratifs concernés ${ }^{45}$; si la réalisation des rapports est encore souvent confiée à des cadres de la fonction publique ou à des bureaux d'études, une collaboration s'amorce depuis peu avec les différents acteurs locaux, et notamment avec les Universités ${ }^{46}$. Dans certains cas, l'évaluation est d'ailleurs perçue comme un exercice global et comme une procédure " normale " de gestion publique : les agents concernés font abstraction de la provenance des financements ou de la nature des programmes; une confusion s'établit alors entre les fonds structurels, les contrats passés avec l'Etat et les initiatives locales ${ }^{47}$. On peut donc estimer que l'influence communautaire devient plus floue, plus implicite, mais aussi que le " transfert " a réussi. Cette adaptation progressive des échelons territoriaux s'explique avant tout comme une façon de rivaliser avec l'Etat central en matière de production d'expertise. Comme le constataient Alain Faure et Andy Smith, un " impressionnant bras de fer " s'est engagé au cours des premières années de la décentralisation pour " quantifier et qualifier les implications gestionnaires et électorales des mutations politicoadministratives en cours "48. Depuis une dizaine d'années, certaines autorités locales chercheraient notamment à utiliser l'évaluation pour consolider une légitimité encore embryonnaire $^{49}$ : cette hypothèse se vérifie en particulier en Rhône-Alpes, où l'Assemblée régionale a impulsé en 1991 la mise en place d'un dispositif d'évaluation ambitieux. A la lumière de ce type d'expérience, le CSE national n'hésitait pas à conclure que " l'évaluation est maintenant devenue l'une des composantes normales du fonctionnement de l'institution régionale ${ }^{150}$. Dans le secteur scientifique et technologique, on observe aussi depuis quelques années une multiplication des initiatives en matière d'évaluation, mais cette fois au sein de l'administration centrale. Peu après sa création en 1981, le Ministère de la Recherche et de la Technologie s'est par exemple doté d'un Centre de Prospective et d'Evaluation, dont les responsables ont vite noué des relations étroites avec l'unité bruxelloise chargée du programme FAST. L'évaluation des actions nationales a été imposée comme un impératif par la loi d'orientation et de programmation de la recherche de 1985 ; régulièrement réaffirmé par la suite, cet impératif s'est notamment concrétisé par la création d'un Comité National d'Evaluation de la Recherche et d'un Observatoire des Sciences et des Techniques ${ }^{51}$. Cette évolution peut tout d'abord être perçue comme le produit d'un désir de contrôler et de maîtriser l'intervention communautaire: officiellement favorables à la mise en place à Bruxelles d'une vraie politique de recherche, mais longtemps méfiants face à l'émergence d'une nouvelle instance qui pourrait les déposséder de leurs prérogatives, les agents nationaux auraient finalement choisi de se " battre avec les mêmes armes ". Esquissée par Jacques Toulemonde ${ }^{52}$, cette hypothèse semble étayée par la réalisation en 1992 d'une " contre-évaluation " du second Programme-cadre de recherche communautaire ${ }^{53}$. Mais, en se dotant d'une capacité d'expertise et d'évaluation propre, les agents de l'administration de la recherche ont 
peut-être surtout voulu renforcer leur compétence et leur autorité face à des organismes publics forts. De création récente, soumis à des restructurations majeures à chaque alternance gouvernementale, le Ministère de la Recherche semble avoir eu quelques difficultés à trouver sa place. Sa tutelle est encore contestée par des scientifiques jaloux de leur autonomie: " le Ministère est composé de personnes détachées des organismes de recherche et il faut supposer qu'elles apportent l'expertise suffisante, mais finalement, les véritables acteurs de la recherche, (....) ce sont quand même les organismes "; " il y a une idée-force, qui veut que la science corresponde aux besoins des chercheurs et qu'elle ne soit pas seulement l'affaire des apparatchiks "... ${ }^{54}$ Finalement, il faut constater que les scientifiques eux-mêmes constituent le pôle le plus hostile à la généralisation des méthodes d'évaluation bruxelloises. Les acteurs qui se présentent comme des représentants de la " communauté scientifique " ont en effet leurs propres outils d'expertise et leurs propres règles en matière d'appréciation des travaux de recherche, de recrutement et de gestion des carrières, de sélection des publications, d'attribution des prix et distinctions. Considérés comme universels, ces outils et ces règles ont été exploités par les autorités publiques. Mais, comme le soulignait notamment Christiane RestierMelleray ${ }^{55}$, les scientifiques restent attachés à leurs traditions et ont toujours eu du mal à accepter que la recherche soit évaluée de l'extérieur, comme n'importe quel autre secteur de politique. Dans un rapport réalisé pour la Commission, Robert Chabbal (physicien et ancien Directeur général du CNRS) rappelait par exemple que les scientifiques refusaient que les choix les concernant soient faits par des tiers; tout en reconnaissant l'importance d'une évaluation négociée, associant les représentants des différents intérêts économiques et sociaux, il proposait d'instituer au sein du dispositif d'évaluation de la DGXII un " garant ", qui aurait pour fonction de veiller à l'indépendance des évaluateurs, à l'objectivité et à la rigueur de leurs méthodes ${ }^{56}$. En l'occurrence, l'adaptation a donc peut-être joué en sens inverse : si les services de la Commission prônent comme on l'a vu une évaluation pluraliste, ils continuent dans certains secteurs à faire appel à des scientifiques de renom; la crédibilité et l'acceptabilité des rapports restent encore subordonnées au choix des " experts " ${ }^{57}$. En somme, la construction européenne n'est pas étrangère à l'évolution de la réflexion et des pratiques nationales, notamment françaises, en matière d'évaluation. Mais le processus d'adaptation n'est ni uniforme, ni unilatéral ; par ailleurs, dans ce domaine comme dans beaucoup d'autres, il reste difficile de savoir dans quelle mesure l'Europe a servi de " cause " ou de " prétexte ". Dans les secteurs où elle s'est développée, l'évaluation contribue à provoquer une interaction régulière entre des acteurs représentant des niveaux de Gouvernement distincts et des sphères économiques et sociales multiples; elle offre un cadre dans lequel peuvent s'opérer une confrontation ou une convergence des critères de perception et des valeurs, des formes d'expertise et des intérêts. Au bout du compte, un modèle spécifique tend à s'imposer; un " changement " relatif s'opère au sein du système européen... Il n'est toutefois pas question ici de raisonner en termes de " rupture " ou de " seuil qualitatif "58. Le recours à l'évaluation, définie sous sa forme " pluraliste ", peut certes commencer à se généraliser à partir du moment précis où il devient une condition à l'obtention des crédits publics. Mais contrairement à ce qu'on a pu constater dans d'autres domaines ${ }^{59}$, la diffusion d'un modèle communautaire de l'évaluation constitue un phénomène trop flou et trop indirect pour être la cause d'une crise ou d'une contestation ouvertes; les enjeux sont difficiles à discerner, le cercle des participants est restreint et aucun 
groupe ne s'est érigé en " traducteur " ou en " porte-parole " des oppositions éventuelles. En tout état de cause, les normes communautaires peuvent se superposer sans les atteindre vraiment à d'autres normes plus profondément ancrées : toutes les formes $\mathrm{d}^{\prime \prime}$ habillage ", de réinterprétation ou de détournement restent envisageables; l'adaptation ne peut être que progressive et consentie (quelles que soient les raisons réelles du consentement). En d'autres termes, on peut se convertir à une certaine pratique de l'évaluation sans abjurer sa foi antérieure: le système communautaire pourrait bien se caractériser avant tout par son syncrétisme. Mais l'évolution qui se dessine n'est pas sans risque. Même si elles se heurtent à des formes de résistance diffuses, ou si elles ne suscitent que des adhésions d'opportunité, les pratiques communautaires continuent d'être justifiées par leur efficacité objective: en prétendant implanter dans les Etats membres des méthodes de " management " à vocation universelle, au lieu de revendiquer ce qui constitue en dernier ressort un choix politique, les autorités bruxelloises esquivent le débat public en même temps qu'elles évitent le conflit. Par ailleurs, le discours qui consiste à présenter l'évaluation comme un moyen privilégié de " rendre compte " aux citoyens et la propension de la plupart des instances impliquées à utiliser cet instrument pour renforcer leur légitimité sont difficilement acceptables, puisqu'en définitive ce ne sont pas les gouvernés en tant que tels qui posent les questions et qui définissent les critères de jugement sur l'action publique.

\section{NOTES}

1. Maître de Conférences en science politique à l'Université d'Amiens et membre du Centre de Recherche Universitaire sur la Construction Européenne (CRUCE).

2. Conseil Scientifique de l'Evaluation français.

3. Perret B., " L'évaluation des politiques publiques comme procédure d'intégration de la connaissance sociale au fonctionnement des systèmes administratifs et politiques ", Bruxelles, 27 mars 1996 (cité par Leca J. lors du Colloque international sur "

L'évaluation des politiques publiques " organisé par le Groupe d'Analyse des Politiques Publiques à Cachan les 5 et 6 février 1997).

4. Pour reprendre les termes de Leca J. (intervention au Colloque international sur " L'évaluation des politiques publiques ", op. cit..

5. Le découpage séquentiel idéal fait intervenir l'évaluation entre la " mise en œuvre sur le terrain " et la " terminaison " des politiques publiques (voir Jones C. O., An introduction to the study of public policy, Belmont, Luxbury Press, 1970).

6. Voir dans ce sens Kluckhohn C., " Values and value-orientation on the theory of action " in Parsons T. et Shils E. (eds), Toward a general theory of action, Harvard University Press, 1951.

7. OCDE, L'évaluation des programmes d'innovation technologique, Paris, 1988.

8. Deleau M. (dir.), Evaluer les politiques publiques, rapport pour le Commissariat Général au Plan, Paris, la Documentation française, 1986. 
9. Nioche J. P., " De l'évaluation à l'analyse des politiques publiques ", Revue française de Science Politique, vol. 32, $\mathrm{n}^{\circ} 1$, février 1982 ; Nioche J. P. et Poinsart R., L'évaluation des politiques publiques, Paris, Economica, 1984.

10. Viveret P., L'évaluation des politiques et des actions publiques, Rapport au Premier Ministre, Paris, la Documentation française, 1989.

11. Monnier E., Evaluation de l'action des pouvoirs publics, du projet au bilan, Paris, Economica, 1987 ; et " L'évaluation dans le triangle de la décision : opinions singulières et processus pluraliste ", Politiques et management public, vol. 10, septembre 1992. 12. Voir dans ce sens Restier-Melleray C., " Experts et expertise scientifique ", Revue française de science politique, $n^{\circ} 4$, août 1990 et " Les ambiguïtés de l'évaluation de la recherche publique en France ", Politiques et management public, vol. 6, n 4 , décembre 1988. Voir également Rouban L., L'Etat et la science ; la politique publique de la science et de la technologie, Paris, CNRS, 1988. Curieusement, certains auteurs défendent la thèse inverse (voir par exemple Lascoume P. et Setbon M., " L'évaluation pluraliste des politiques publiques, enjeux, pratiques, produits ", rapport préparé pour le Commissariat Général au Plan, janvier 1996).

13. On peut noter que ce décret s'efforce de trancher entre les conceptions en présence pour donner une définition officielle de l'évaluation : " évaluer une politique, c'est rechercher si les moyens juridiques, administratifs ou financiers mis en œuvre permettent de produire les effets attendus de cette politique et d'atteindre les objectifs qui lui sont fixés ".

14. Leca J., intervention au Colloque international sur " L'évaluation des politiques publiques ", op. cit..

15. Article 130 F.1 du traité CEE modifié par le traité sur l'Union européenne.

16. JOC. 7 du 29/1/74.

17. En 1978, il a été intégré dans le programme FAST (Forecasting and Assessment in the Field of Science and Technology - Prospective et évaluation dans le domaine de la science et de la technologie), doté de 4,4 millions d'ECU pour la période 1978-1982, puis de 8,5 millions pour 1983-1987 ; par la suite, FAST a lui-même été fondu dans le programme MONITOR (Strategic Analysis, Forecasting and Evaluation in Matters of Research and Technology - Analyse stratégique, prospective et évaluation en matière de recherche et de technologie, 1989-1993, 22 millions d'ECU), puis dans un programme de " recherche socio-économique finalisée " (1994-1998, 138 millions d'ECU).

18. Voir notamment les résolutions du Conseil relatives à un " cadre pour l'évaluation des programmes de recherche et de développement " (28 juin 1983, JOC213 du 9/8/83) et à un " plan d'action communautaire pour l'évaluation " (9 décembre 1986, JOC2 du $6 / 1 / 87$ ). A partir de 1988, les règles relatives à l'évaluation ont été fixées dans le cadre des programmes MONITOR puis " recherche socio-économique finalisée ".

19. Selon les termes de plusieurs responsables de la Commission.

20. Pour reprendre les conclusions de Toulemonde J., " Partages de souveraineté en matière de politique scientifique : que révèlent les évaluations ? ", communication au Colloque international organisé par la revue Politiques et Management public et l'Université Libre de Bruxelles, " la souveraineté éclatée ", Bruxelles, 11 et 12 octobre 1990.

21. Article $130 \mathrm{~A}$ du traité CEE modifié par le traité sur l'Union européenne.

22. Règlement-cadre du 24 juin 1988 (JOL185 du 15/7/88) et règlements du 19 décembre 1988 (JOL374 du 31/12/88).

23. JOL193 du 31/7/93. 
24. Selon les responsables de l'unité d'évaluation, ce chiffre volontairement restreint permettrait d'échapper au critère de représentativité nationale ; dans la pratique, il semblerait toutefois qu'un " dosage " entre les ressortissants des " petits " et "grands " pays soit assuré.

25. Bobe B. et Viala H., Une décennie d'évaluation de la R\&D à la Commission européenne, Bruxelles, Ecole Centrale / Commission des Communautés européennes, 1990.

26. Voir notamment les " Orientations communes pour le suivi et les évaluations intermédiaires ", Commission européenne, Bruxelles, 1995.

27. La DGXIII (" Télécommunications, Marché de l'Information et Valorisation de la Recherche "), qui partage avec la DGXII la gestion de certains programmes technologiques, possède également une unité " Evaluation et Valorisation des résultats de la recherche ". La DGII (" Affaires économiques et financières ") s'est quant à elle dotée de plusieurs unités chargées de l'évaluation des fonds structurels, des politiques sociales et agricoles, des politiques de la concurrence, de la recherche, de l'environnement, des transports et de l'énergie.

28. Le secteur de la coopération au développement n'a pour l'instant fait l'objet que d'un nombre très limité de recherches et les rapports publiés à Bruxelles restent trop nombreux et hétérogènes pour qu'on puisse à ce stade réaliser une synthèse cohérente. Cet article n'y fera donc pas référence ; une étude plus approfondie serait néanmoins opportune.

29. Voir sur ce point Majone G., La Communauté européenne : un Etat régulateur, Paris, Monchrestien, 1996.

30. " Evaluation finale du second Programme-cadre de recherche et développement communautaire ", communication présentée par la Commission au Conseil et au Parlement européen le 22 avril 1992 (COM(92)675).

31. Callon M. et Laredo P., L'impact des programmes communautaires sur le tissu scientifique et technologique français, Paris, la Documentation française, 1990.

32. European Strategic Programme for Research in Information Technology (Programme stratégique européen pour la recherche dans les technologies de l'information).

33. " Rapport d'évaluation à mi-parcours du programme ESPRIT ", Commission des Communautés européennes, Bruxelles, novembre 1985.

34. Entretiens avec des responsables scientifiques ou industriels.

35. Damiani A., " Tendances de la recherche en Europe : le Programme-cadre est adopté ", Revue du Marché Commun, n³08, juillet 1987.

36. Salomon J. J., allocation à la conférence annuelle de l'Association Européenne de Gestion de la Recherche Industrielle, Bruxelles, 31 mai-2 juin 1989.

37. University of Manchester, The impact and utility of European Community research programmes evaluation reports, Bruxelles, Commission des Communautés européennes, 1990.

38. Entretiens avec des responsables industriels et des dirigeants d'organismes de recherche français.

39. Une décennie d'évaluation de la R\&D à la Commission européenne, op. cit..

40. Sur ce point, voir notamment Le Naëlou A., Politiques européennes de développement avec les pays du Sud, Paris, L'Harmattan, 1995, Jourdain L., Recherche scientifique et construction européenne, Paris, L'Harmattan, 1995, Balme R., " La politique régionale communautaire comme construction institutionnelle " in Politiques 
publiques en Europe, dir. par Mény Y., Müller P. et Quermonne J. L., Paris, L'Harmattan, 1995 ou Smith A., L'Europe au miroir du local, Paris, L'Harmattan, 1996.

41. Pour reprendre les termes de Monnier E., Degeorges O. et Spenlehauer V., " L'évaluation comme outil de légitimation : le cas des grandes organisations internationales ", Politiques et Management public, décembre 1990, vol. 8, n4. Les auteurs comparent en l'occurrence les pratiques de la Communauté Européenne, de l'ONU et de la Banque Mondiale.

42. Dans le cas de la France, on peut citer l'Ecole Centrale, le Centre " Science, Technologie et Société " du Conservatoire National des Arts et Métiers ou encore le Centre de Sociologie de l'Innovation de l'Ecole des Mines.

43. Ces relais nationaux semblent d'ailleurs reproduire le discours, les méthodes et les arguments communautaires. Dans sa note précitée de mai 1995 (voir supra), la DATAR insistait par exemple sur le fait que l'évaluation n'avait pas pour objet de " sanctionner ou contrôler tel et tel gestionnaire ", mais de " rendre plus efficace l'utilisation des crédits publics ". Elle ajoutait que l'évaluation représentait désormais en France " une priorité gouvernementale ", en même temps qu'un " sujet complexe et mal connu exigeant un effort d'explication particulier ".

44. On peut noter que ce cabinet a été créé par E. Monnier, voir supra.

45. La situation se présente bien sûr différemment d'une région à l'autre. A. Smith, E. Monnier et P. Duran ont par exemple constaté qu'en Bretagne ou dans le Nord-Pas de Calais, le " partenariat " devenait la norme dominante de l'action publique locale, tandis qu'en Rhône-Alpes, la tension existant entre les différents échelons territoriaux (notamment entre le département et la région) faisait de l'évaluation un objet de conflit in " Evaluation à la française ", Evaluation, vol. 1, n¹ , 1995.

46. C'est le cas depuis quelques années en Bretagne, en Bourgogne ou, plus récemment, en Picardie.

47. Cette confusion apparaît par exemple dans le discours des responsables du Secrétariat Général à l'Action Régionale de Picardie ; elle est de plus en plus manifeste, au fur et à mesure, qu'on descend vers des échelons territoriaux plus petits (entretiens menés à l'occasion de l'évaluation intermédiaire du Document Unique de Programmation relatif à l'objectif 2 des fonds structurels en Picardie).

48. " L'évaluation objet de savoir et enjeu de pouvoir ", Sciences de la société, $n^{\circ} 32$, mai 1994.

49. Voir également dans ce sens Smith A., Monnier E. et Duran P., " Evaluation à la française ", op. cit..

50. Rapport annuel du Conseil Scientifique de l'Evaluation sur l'évolution des pratiques d'évaluation des politiques publiques en France, Paris, la Documentation française, 1995.

51. Il faut également rappeler l'existence en France, depuis 1983, d'un Office Parlementaire d'Evaluation des Choix Scientifiques et Technologiques, plus ou moins conçu sur le modèle de l'Office of Technology Assessment du Congrès américain ; en 1985, le Parlement européen s'est d'ailleurs doté à son tour d'un Office Parlementaire d'Evaluation des Choix Scientifiques et Techniques.

52. Communication au Colloque international organisé par la revue Politiques et Management public et l'Université Libre de Bruxelles, op. cit..

53. Critiques face aux conclusions des experts mandatés par la Commission (voir supra) et désireux de ne pas laisser aux services bruxellois le monopole de l'évaluation, les représentants du Ministère de la Recherche et de la Représentation permanente 
française ont en effet incité leurs homologues étrangers à piloter une seconde évaluation, par le biais du Comité de la Recherche Scientifique et Technique (CREST), dont ils faisaient partie.

54. Entretiens avec des responsables d'organismes publics de recherche.

55. " Experts et expertise scientifique " et " Les ambiguïtés de l'évaluation de la recherche publique en France ", op. cit..

56. Organization of research evaluation in the European Community, Bruxelles, OCDE / Commission des Communautés européennes, 1990 (R. Chabbal était à la tête de la Direction pour la science, la technologie et l'industrie de l'OCDE au moment de la rédaction de ce rapport).

57. Les personnes destinées à représenter le " monde de l'industrie " au sein des panels d'évaluation sont souvent choisies parce qu'elles bénéficient d'une autre source de légitimité : le Président du comité chargé de l'évaluation à mi-parcours du second Programme-cadre était par exemple conseiller à la présidence du groupe Thomson, mais il s'agissait surtout d'un ancien chercheur et d'un ancien Secrétaire d'Etat à la Recherche, membre de l'Académie des Sciences.

58. Voir dans ce numéro l'article de Rouban L.

59. Idem.

INDEX

Index géographique : Bruxelles, Europe, Union européenne

Mots-clés : politiques publiques, construction européenne, bureaucraties/ théorie des organisations 